\title{
Fetoscopic laser photocoagulation in twin-to-twin transfusion syndrome: experience from a single institution
}

Edwin $\underline{T h i a}^{1}$, MBBS, MRCOG, Serene $\underline{T h a i n}^{2}$, MBBS, MRCOG, George SH $\underline{Y e O}^{1}$, MBBS, FRCOG

INTRODUCTION Twin-to-twin transfusion syndrome (TTTS) is the most common serious complication of monochorionic (MC) twin pregnancies, with perinatal mortality rates of up to $90 \%$ if untreated. This study aimed to review the perinatal and perioperative outcomes of MC twin pregnancies treated for TTTS by fetoscopic laser photocoagulation (FLP) since its introduction at KK Women's and Children's Hospital (KKH), Singapore, in 2011.

METHODS This was a retrospective review of five consecutive patients who underwent FLP of placental anastomoses for TTTS at KKH from June 2011 to March 2014. FLP was offered to patients who were diagnosed with TTTS of at least Quintero Stage II before 26 weeks of gestation. The main outcome measures were perioperative complications and perinatal survival rates.

RESULTS Five sets of MC twin pregnancies underwent FLP during the study period - three pregnancies were diagnosed with Stage III TTTS and two pregnancies with Stage II TTTS. Median gestational ages at initial presentation, laser photocoagulation and delivery were 19 (range 17-20) weeks, 20 (range 19-23) weeks and 29 (range 28-34) weeks, respectively. One patient had bleeding into the amniotic cavity intraprocedurally. Overall, the perinatal survival rate, double-infant survival rate and survival rate for at least one twin were $60 \%$ (6/10 fetuses), $40 \%(2 / 5$ twins) and $80 \%$ (4/5 twins), respectively.

CONCLUSION FLP is a feasible treatment for TTTS, with minimal maternal complications. Perinatal survival rates of this patient group that was managed at our centre were comparable to those of international centres.

Keywords: fetofetal transfusion, fetoscopic laser photocoagulation, monochorionic

\section{INTRODUCTION}

Monochorionic (MC) twin pregnancies arise from the cleavage of a single fertilised egg after the third day of fertilisation. This occurs in about two-thirds of all monozygous twin pregnancies. Twinto-twin transfusion syndrome (TTTS) is the single most common serious complication of MC twin pregnancies, occurring in 15\% of these pregnancies and thus affecting one in 1,600 pregnancies. ${ }^{(1)}$ Untreated TTTS has very poor prognosis, with perinatal mortality rates of up to $90 \%$ and the donor being at higher risk of death when compared to the recipient. ${ }^{(2)}$

There is now extensive ex vivo and in utero evidence to show that TTTS results from a chronic, imbalanced unidirectional blood flow between the twins, i.e. from artery to vein, through placental deep arteriovenous anastomoses (AVAs) between the two fetal circulations sharing the same placenta mass. ${ }^{(3,4)}$ These AVAs are present in $90 \%$ of $\mathrm{MC}$ placentae. As a result of the imbalance of blood flow from one twin to the other, the donor twin shows signs of hypovolaemia and growth discordance with oliguria, oligohydramnios and abnormal findings on umbilical artery (UA) Doppler ultrasonography. The recipient twin shows signs of overload with polyhydramnios, cardiomegaly and abnormal findings on venous Doppler ultrasonography (e.g. reverse flow in the ductus venosus [DV] or pulsatile flow within the umbilical vein) and, in extreme circumstances, hydrops.
Fetoscopic laser photocoagulation (FLP) is one of the current treatments for TTTS that has come about in recent years, and is the only intervention that targets the aforementioned pathophysiology of TTTS. It has been shown to be a more effective first-line treatment than serial amnioreduction for severe TTTS diagnosed before 26 weeks of gestation, as demonstrated in the Eurofetus study. ${ }^{(5)}$ We first introduced the FLP procedure for treatment of TTTS at KK Women's and Children's Hospital (KKH), Singapore, in June 2011. The aim of this study was to review the perioperative and perinatal outcomes of our initial experience with FLP.

\section{METHODS}

The present study was a retrospective case series review of the five patients who underwent FLP at our unit from June 2011 to March 2014. The FLP procedure was first introduced at KKH in June 2011. Prior to the implementation of this service, training and workshops for FLP were conducted from March 2010. FLP was offered only to patients with pregnancies complicated by TTTS of at least Quintero Stage II and before 26 weeks of gestation. All the procedures were performed by a trained and experienced operator. Patients were admitted on the day of the procedure and discharged the following day if all was well. They were then followed up closely in the Fetal Medicine Clinic at least once weekly or twice a week, to be monitored

${ }^{1}$ Department of Maternal Fetal Medicine, ${ }^{2}$ Department of Obstetrics and Gynaecology, KK Women's and Children's Hospital, Singapore 
Table I. Antenatal characteristics of patients who underwent FLP for twin-to-twin transfusion syndrome.

\begin{tabular}{|c|c|c|c|c|c|}
\hline Characteristic & Patient 1 & Patient 2 & Patient 3 & Patient 4 & Patient 5 \\
\hline Maternal age (yr) & 34 & 35 & 32 & 41 & 28 \\
\hline Gestation at diagnosis (wk) & 19 & 20 & 17 & 20 & $19+2$ \\
\hline Quintero stage at diagnosis & III & III & III & II & II \\
\hline Gestation at FLP (wk) & $19+2$ & $23+2$ & 21 & $20+3$ & $19+3$ \\
\hline Gestation at delivery (wk) & $30+6$ & 29 & $27+4$ & $28+5$ & 34 \\
\hline
\end{tabular}

FLP: fetoscopic laser photocoagulation; IUD: intrauterine death; LSCS: lower-segment Caesarean section; VD: vaginal delivery

for evidence of resolution, persistence or recurrence of TTTS. Parameters measured included deepest vertical pool, presence or absence of fetal bladder, standard deviation score of abdominal circumference, and Doppler studies of the UA, DV and middle cerebral artery (MCA). Operative details, perioperative and postoperative complications, and pregnancy outcomes were reviewed.

The overall survival rate was calculated by taking the number of surviving fetuses divided by the total number $(n=10)$ of fetuses. The double-infant survival rate was the proportion of pregnancies in which both fetuses survived. The one-twin survival rate was the proportion of pregnancies in which one or both fetuses survived.

All procedures were performed in the Antenatal Diagnostic Centre of $\mathrm{KKH}$. All the patients had local anaesthesia for the procedure. Antibiotic prophylaxis in the form of intravenous cefazolin $1 \mathrm{~g}$ was given to all patients 30 minutes before the procedure. An ultrasonography examination was performed prior to the procedure to confirm the viability of the twin pregnancy, determine the location of the placenta and cord insertions of both twins, and to plan for the site of entry of the fetoscope. Instruments used included a 2-mm fetoscope (HOPKINS ${ }^{\text {II }}$ Straight Forward Telescope $0^{\circ}$ 26008AA; Karl Storz, Tuttlingen, Germany), a diode laser with a 600- $\mu \mathrm{m}$ laser fibre (Dornier Medilas D MultiBeam; Dornier MedTech, Wessling, Germany), vascular access trocar and cannula (Terumo Radifocus 11F; Terumo Corporation, Tokyo, Japan). Under ultrasonographic guidance, the trocar and cannula were inserted into the amniotic cavity of the recipient twin using the Seldinger method after a small abdominal incision was made. The site of entry was dependent on the placental location as well as the placental cord insertions of both twins. Once inside the amniotic cavity, anastomotic vessels crossing between the twins (vascular equator) were selectively identified and coagulated using the laser at $30 \mathrm{~W}$, as in the method described by Quintero et $\mathrm{al}^{(6)}$ in 2001. If amnio-infusion was deemed necessary to maintain adequate vision within the amniotic cavity, warm Hartmann's solution via a side port of the trocar was used. At the end of the procedure, amnioreduction was performed to reduce the amount of amniotic fluid in the recipient sac to that of normal liquor volume. Fetal viability was confirmed with ultrasonography at the end of the procedure, and Doppler studies of the UA, DV and MCA of both twins were routinely performed. The patient was then admitted for an overnight stay and started on oral nifedipine $10 \mathrm{mg}$ administered four times a day.
Table II. Perinatal outcomes of the five monochorionic-diamniotic twin pregnancies treated with fetoscopic laser photocoagulation for twin-to-twin transfusion syndrome.

\begin{tabular}{lc}
\hline Perinatal outcome & No. (\%) \\
\hline Overall survivor (per fetus) $(n=10)$ & $6(60)$ \\
Double survivor (per pregnancy) $(n=5)$ & $2(40)$ \\
Only one survivor (per pregnancy) $(n=5)$ & $1(20)$ \\
At least one survivor (per pregnancy) $(n=5)$ & $4(80)$ \\
Gestational age at delivery $(w k)^{*}$ & $29(28-34)$ \\
Procedure-delivery interval $(w k)^{*}$ & $8(6-15)$ \\
\hline
\end{tabular}

${ }^{*}$ Data presented as median (range).

\section{RESULTS}

A total of five patients who underwent FLP from June 2011 to March 2014 were reviewed in this study. Four of the five patients were referred to our institution after 19 weeks of gestation. Of the four referred patients, three were booked early in private local hospitals, while one was booked early in an overseas hospital. The fifth patient was booked early in our institution. Antenatal characteristics of the five patients are shown in Table I. The median gestational age at presentation was 19 (range 17-20) weeks, and the median gestational age at laser photocoagulation was 20 (range 19-23) weeks. The severity of TTTS at the time of the procedure was Stage III for $3(60 \%)$ patients and Stage II for the remaining 2 (40\%) patients.

Perinatal outcomes and delivery details are summarised in Table II. The median interval between laser treatment and delivery was 8 (range 6-15) weeks. There was one case of bleeding into the amniotic cavity as a result of haematoma formation at the uterineplacenta wall insertion site during the fetoscopic procedure. The overall fetal survival rate was $60 \%$ (6/10 fetuses). There was at least one surviving infant in $80 \%$ (4/5 twins) of the treated pregnancies. The double-infant survival rate was $40 \%$ (2/5 twins). The median gestational age at delivery was 29 (range 28-34) weeks. Neonatal outcomes are shown in Table III, and the five MC twin pregnancies are described individually in greater detail.

\section{Patient 1}

A 34-year-old woman (gravida 4, para 0) with a history of three previous miscarriages was booked early with a private obstetrician initially. She was referred to our institution at 19 weeks of gestation for possible TTTS and was later diagnosed with Stage III TTTS at our centre. FLP was performed at $19+2$ weeks of gestation, according to our local protocol. TTTS resolved after FLP and both twins continued to grow till $30+2$ weeks. The ex-donor twin 
Table III. Neonatal outcomes of the five monochorionic-diamniotic twin pregnancies treated with fetoscopic laser photocoagulation for twin-to-twin transfusion syndrome.

\begin{tabular}{|c|c|c|c|c|c|}
\hline Characteristic & Patient 1 & Patient 2 & Patient 3 & Patient 4 & Patient 5 \\
\hline $\begin{array}{l}\text { Birth weight }(\mathrm{g}) \text { (donor/ } \\
\text { recipient) }\end{array}$ & $\begin{array}{l}-/ 1,505 \\
\text { (donor IUD) }\end{array}$ & $\begin{array}{l}398 / 760 \\
\text { (both IUD) }\end{array}$ & $773 / 1,224$ & $\begin{array}{l}\text { 780/- } \\
\text { (recipient IUD) }\end{array}$ & $1,200 / 2,000$ \\
\hline \multicolumn{6}{|l|}{$\begin{array}{l}\text { Apgar score (donor/ } \\
\text { recipient) }\end{array}$} \\
\hline At $1 \mathrm{~min}$ & -19 & - & $5 / 9$ & $8 /-$ & NA \\
\hline At $5 \mathrm{~min}$ & -19 & - & $8 / 9$ & $8 /-$ & NA \\
\hline NICU admission & One twin & - & Both twins & One twin & NA \\
\hline Survival up to 28 days & One twin & - & One twin & One twin & Both twins \\
\hline $\begin{array}{l}\text { NICU stay (day) (donor/ } \\
\text { recipient) }\end{array}$ & $-/ 13$ & - & $17 /-$ & - & NA \\
\hline $\begin{array}{l}\text { LOS (day) (donor/ } \\
\text { recipient) }\end{array}$ & $-/ 41$ & - & $-/ 76$ & $175 /-$ & NA \\
\hline Perinatal morbidity & $\begin{array}{l}\text { Recipient: hypoglycaemia, } \\
\text { NNJ, mild pulmonary } \\
\text { stenosis, late-onset } \\
\text { Group B streptococcal } \\
\text { sepsis }\end{array}$ & - & $\begin{array}{l}\text { Donor: hypoglycaemia, } \\
\text { NNJ, RDS, PPHN, PDA, } \\
\text { VSD, IVH, ARDS (demised } \\
\text { after } 17 \text { days of life) } \\
\text { Recipient: hypoglycaemia, } \\
\text { RDS, PDA, IVH, ROP, NNJ }\end{array}$ & $\begin{array}{l}\text { Donor: hyaline } \\
\text { membrane disease, } \\
\text { PDA, sepsis, NNJ, } \\
\text { ileal stenosis } \\
\text { with intestinal } \\
\text { obstruction } \\
\text { necessitating } \\
\text { ileostomy, infantile- } \\
\text { type PCKD }\end{array}$ & NA \\
\hline
\end{tabular}

ARDS: acute respiratory distress syndrome; IUD: intrauterine death; IVH: intraventricular haemorrhage; LOS: length of hospital stay; NA: not available; NICU: neonatal intensive care unit; NNJ: neonatal jaundice; PCKD: polycystic kidney disease; PDA: patent ductus arteriosus; PPHN: persistent pulmonary hypertension of the newborn; RDS: respiratory distress syndrome; ROP: retinopathy of prematurity; VSD: ventricular septal defect

suffered an unexpected intrauterine fetal death at $30+2$ weeks (Day 77 post-FLP). Serial measurements of MCA-peak systolic velocity (PSV) were performed daily to monitor the status of the ex-recipient twin; the measurements were noted to be on a consistently increasing trend, from 1.11 multiples of median (MoM) to 1.20 MoM, and finally at 1.29 MoM over the three days following the demise of the ex-donor twin. Decision for delivery via elective Caesarean section at $30+6$ weeks was made in view of the rising MCA-PSV in the ex-recipient twin, which indicated possible onset of fetal anaemia. Birth weight of the surviving twin was 1,505 $\mathrm{g}$ and the Apgar scores were the same at one and five minutes (i.e. 9). Haemoglobin level of the neonate at birth was normal, at $18.6 \mathrm{~g} / \mathrm{dL}$. The baby stayed in the neonatal intensive care unit for 13 days and the special care nursery for 28 days, and was discharged from hospital on Day 41 of life.

\section{Patient 2}

A 35-year-old woman (gravida 1, para 0) with a spontaneous monochorionic-diamniotic (MCDA) twin pregnancy was booked early at an overseas hospital initially. She was diagnosed with TTTS at 20 weeks of gestation. The patient decided to seek treatment at our institution and was booked at 23 weeks. She was diagnosed with Stage III TTTS at our centre and FLP was performed at $23+2$ weeks. Laser ablation was performed on the identified anastomotic vessels. The procedure was complicated by haematoma formation at uterine-placenta wall insertion intraoperatively, and the liquor became bloodstained as a result. Laser fetoscopy was thereafter terminated and amnioreduction was performed, with 2,000 mL of bloodstained liquor reduced in total. The patient was admitted to the labour ward for monitoring.
Repeat ultrasonography the following day noted cessation of fetal cardiac activity of the donor twin. Doppler changes in the recipient resolved and the ex-recipient twin continued to grow. However, the ex-recipient twin died on Day 39 post-FLP at 28 +6 weeks of gestation.

\section{Patient 3}

A 32-year-old woman (gravida 2, para 1) with a history of one previous lower-segment Caesarean section was booked early with a private obstetrician initially. She was diagnosed with TTTS at 17 weeks of gestation and referred to our centre at $20+1$ weeks, where Stage III TTTS was diagnosed and FLP was performed at 21 weeks. TTTS resolved, but the ex-donor twin appeared to have Type 3 selective intrauterine growth restriction as defined by Gratacós et al, ${ }^{(7-9)}$ with the estimated fetal weight of the small fetus falling below the tenth percentile, and the presence of intermittent absent and reversed end-diastolic flow on UA Doppler ultrasonography. She was monitored closely in view of the abnormal Doppler changes. At $27+2$ weeks of gestation, the ex-donor twin had reversed a-wave noted on DV Doppler ultrasonography and hence a decision was made for delivery. Intramuscular dexamethasone was administered and the patient delivered via elective Caesarean section at $27+4$ weeks of gestation. Birth weights of the ex-recipient and ex-donor twins were 1,224 g and $773 \mathrm{~g}$, respectively. The Apgar scores at one and five minutes for the ex-recipient and ex-donor twins were 9 and 9, and 5 and 8, respectively. The ex-recipient twin was discharged on Day 76 of life. However, the ex-donor twin died on Day 17 of life due to sepsis and prematurity. 


\section{Patient 4}

A 41-year-old woman (gravida 6, para 2) with a history of three previous miscarriages and two previous normal vaginal deliveries was booked early with a private obstetrician. This was a spontaneous MCDA twin pregnancy. She was diagnosed with TTTS at 20 weeks of gestation and referred to our centre the day after diagnosis. Stage II TTTS was diagnosed and FLP was performed at $20+3$ weeks of gestation. However, TTTS persisted despite the performance of FLP; the donor twin continued to have an empty bladder on follow-up visits. The mother then underwent amnioreduction at $23+1$ weeks of gestation. However, as there was no improvement post-amnioreduction, repeat FLP was performed at $24+3$ weeks of gestation. Repeat FLP is one of the approaches taken for the management of recurrent TTTS (other options include amnioreduction, expectant management or cord occlusion), with the internationally reported rate of a repeat procedure being $29.6 \%$ according to a systematic review in 2012. ${ }^{(10)}$ However, the ex-recipient twin died two days after repeat FLP at $24+5$ weeks (Day 30 post-first FLP). The ex-donor twin survived and its amniotic fluid volume improved, with visualisation of the bladder. The patient subsequently went into spontaneous preterm labour at $28+5$ weeks of gestation and delivered a baby of birth weight $780 \mathrm{~g}$ and Apgar scores of 8 at one minute and 8 at five minutes. The baby had multiple medical problems but was discharged on Day 175 of life.

\section{Patient 5}

A 28-year-old woman (gravida 1, para 0) had booked early at our institution, at 12 weeks of gestation with a spontaneous MCDA twin pregnancy. She was diagnosed with Stage II TTTS at $19+$ 2 weeks of gestation and underwent FLP the following day at $19+$ 3 weeks of gestation. TTTS was resolved post-FLP and Doppler ultrasonography remained normal. The patient returned to her home country at 25 weeks of gestation and delivered eventually at 34 weeks of gestation via a Caesarean section for preeclampsia and worsening Doppler ultrasonography findings. Birth weights of the ex-donor and ex-recipient twins were 1,200 $\mathrm{g}$ and 2,000 g, respectively. As the patient had delivered at a foreign institution, there was no available data regarding neonatal outcomes, neonatal complications or total length of inpatient neonatal stay.

\section{DISCUSSION}

With the poor prognosis that accompanies TTTS in MC twin pregnancies, it is imperative for clinicians to achieve an early diagnosis so that early intervention may be possible and morbidity may be reduced. Over the last two decades, various interventions for the management of TTTS have been developed in a bid to improve the outcome of such pregnancies. These include serial amnioreduction, inter-twin septostomy, FLP and cord coagulation. All these treatment options have been found to result in improved survival rates compared to untreated TTTS. However, of the various treatment options, FLP (which was first described by De Lia et $\mathrm{al}^{(11)}$ in 1990) is thus far the only treatment aimed at treating the underlying pathophysiology. FLP has been shown to be a more effective first-line treatment than serial amnioreduction for severe TTTS diagnosed before 26 weeks of gestation, as demonstrated in the Eurofetus study. ${ }^{(5)}$ Published series on FLP showed a 55\%-73\% survival rate, with a $4.2 \%$ neurological handicap rate. ${ }^{(12-14)} \mathrm{A}$ Cochrane review in 2008 by Roberts et $\mathrm{al}^{(15)}$ also suggested that FLP of anastomotic vessels should be considered in the treatment of all stages of TTTS to improve perinatal and neonatal outcomes. However, the same review did not show that FLP reduces the risk of neurodevelopmental delay compared with other treatments.

Historically, serial amnioreduction has been one of the key therapies used in the management of TTTS. This intervention involves the repeated removal of large volumes of amniotic fluid, to improve outcome by reducing the risk of preterm labour and delivery secondary to polyhydramnios. It has also been postulated that serial amnioreduction improves fetal haemodynamics by decreasing pressure on the placental surface, thereby allowing increased blood flow to the uterus. Data from the International Amnioreduction Registry shows that at least one fetus survived to four weeks after birth in $71 \%$ of cases and both fetuses survived in $48 \%$ of cases. ${ }^{(16)}$ However, $25 \%$ of these survivors had abnormalities on neonatal cranial ultrasonography. ${ }^{(16)}$

Another therapeutic option for TTTS is cord coagulation using bipolar cautery or radiofrequency ablation, as a form of selective feticide. However, this therapy can only achieve a maximum survival rate of $50 \%$, since the aim is to sacrifice one of the fetuses in a bid to achieve a better prognosis for the remaining twin. In one study of 14 patients, survival rates for the remaining twin after cord coagulation were $80 \%-90 \%$, with no neurological abnormalities evident prior to discharge from hospital. ${ }^{(17)}$ However, this treatment may not be an acceptable option for parents of MC twins unless used as a last resort for pre-terminal TTTS, or if parents desire a better prognosis for one twin as opposed to a guarded prognosis for both.

One other intervention, inter-twin septostomy, which involves the intentional puncture of the inter-twin membrane, was first described by Saade et al to treat TTTS; the procedure has a survival rate of up to $83.3 \% .{ }^{(18)}$ A randomised trial of septostomy versus amnioreduction has shown no difference in overall survival $(70 \%)$; however, there was a decrease in the need for repeat procedures with septostomy $(40 \%$ in the septostomy group vs. $70 \%$ in the amnioreduction group). ${ }^{(19)}$

Despite our study being a small case series owing to the small population base in Singapore, our results showed a $60 \%$ overall survival rate of pregnancies complicated by TTTS treated with FLP. Our results are comparable to those in other published reports. ${ }^{(5,20,21)}$ The severity of TTTS and gestation at delivery are the main factors in determining the perinatal outcome of such pregnancies. ${ }^{(22)}$ It is thus important to diagnose the condition as soon as possible in the second trimester and intervene when appropriate, in order to achieve the best possible outcomes for the MC twin pregnancy. In the present study, the median gestational age at diagnosis was relatively early at 19 weeks, with the median gestational age at intervention (i.e. FLP) at 20 weeks (i.e. a detection-to-intervention interval of only one week). The early diagnosis and intervention in our case series may have 
contributed to the comparable overall survival rates of $60 \%$ found in our patients.

With regard to the technical aspects of FLP in our series, of the five patients, two had anterior placentae and the remaining three had posterior placentae. FLP procedures are known to be more technically challenging in the presence of an anterior placenta, and the approach of scope insertion may need to be altered accordingly to avoid injury and bleeding. For the two patients with anterior placentae, our approach included a detailed pre-fetoscopy ultrasonography mapping of the respective fetal positions, placental location and cord insertions, as well as a more lateral approach for the insertion of the fetoscope so as to avoid the anteriorly located placenta.

In the present study, there was one case of intrauterine death of the donor twin one day after FLP was performed, with the subsequent death of the ex-recipient twin 39 days post-FLP (Patient 2). One possible reason for the death of the donor twin is bleeding complication resulting from the procedure. Another possible reason for the death of the donor twin is unequal placenta sharing, which remained unchanged by the laser procedure. Therefore, after division of the communicating vessels, each fetus relies on the function of its own share of placental mass, which may be insufficient for some fetuses, as was possible in this case. On the other hand, the death of the ex-recipient twin was unlikely to be related to the FLP procedure, as the Doppler changes in the recipient twin had resolved post-FLP, and the ex-recipient twin died only later on Day 39 post-FLP for unidentified reasons.

In our series, single intrauterine death occurred in two of the five patients (Patients 1 and 4). However, there was no evidence of intrauterine demise or neurological handicap in the surviving twin after delivery in both patients. This is likely due to the success of the laser procedure in achieving division of all significant vascular communications between the twins, such that the death of one fetus no longer affected the haemodynamics of the remaining twin. This is one benefit of FLP compared to other available interventions, which do not focus on the separation of vascular communications. Other interventions, such as amnioreduction, do not target the underlying pathophysiology of TTTS and, therefore, the death of one twin would still subject the surviving twin to sudden changes in haemodynamics and an increased risk of intrauterine death or neurological handicap. A review of single intrauterine deaths in MC twin pregnancies showed only a $57 \%$ chance of having a healthy survivor. ${ }^{(23)}$

The median gestational age at delivery in our series was 29 weeks. This is significantly earlier than most other reported large case series. For example, the median gestational age at delivery in the Eurofetus study was 33.3 weeks for FLP compared to 29 weeks for amniodrainage. ${ }^{(5)}$ The earlier median gestational age at delivery for the five patients in our centre may be attributable to a more cautious approach being taken in management in view of our limited experience with laser photocoagulation, as well as the commendable support extended by our hospital's Neonatal Department, which has extensive experience in the management of severely preterm babies and an excellent track record of good neonatal outcomes.
Our study has several limitations. Firstly, the nature of the study, being a retrospective descriptive case series, did not allow us to compare the outcome of TTTS cases treated by FLP versus other methods such as amnioreduction or cord coagulation, which have similarly been used in our institution for the treatment of TTTS. Secondly, long-term neurological and developmental outcomes were not assessed in this case series. Another limitation was the small size of the study population, which is partly due to the small population of Singapore. Despite these limitations, the perinatal outcomes in our series appeared to be comparable to those reported internationally. ${ }^{(24)}$

In conclusion, TTTS can be effectively treated by laser therapy with minimal maternal complications. However, management of this condition requires a specialised multidisciplinary team that is well-versed in the follow-up and detection of complications such as TTTS, and skilled in performing interventional therapies for the treatment of such complications. To achieve the best outcomes, such pregnancies should be managed by maternal fetal medicine specialists in specialised referral centres with tertiary neonatal facilities in order to handle the potential complications of prematurity, which are more likely to occur in these high-risk pregnancies.

\section{REFERENCES}

1. Jain V, Fisk NM. The twin-twin transfusion syndrome. Clin Obstet Gynecol 2004; 47:181-202.

2. El Kateb A, Ville Y. Update on twin-to-twin transfusion syndrome. Best Pract Res Clin Obstet Gynaecol 2008; 22:63-75.

3. Bajoria R, Wigglesworth J, Fisk NM. Angioarchitecture of monochorionic placentas in relation to the twin-twin transfusion syndrome. Am J Obstet Gynaecol 1995; 172:856-63.

4. Denbow ML, Cox P, Taylor M, Hammal DM, Fisk NM. Placental angioarchitecture in monochorionic twin pregnancies: relationship to fetal growth, fetofetal transfusion syndrome, and pregnancy outcome. Am J Obstet Gynaecol 2000; 182:417-26.

5. Senat MV, Deprest J, Boulvain M, et al. Endoscopic laser surgery versus serial amnioreduction for severe twin-to-twin transfusion syndome. N Engl J Med $2004 ; 351: 136-44$

6. Quintero RA, Bornick PW, Allen MH, Johson PK. Selective laser photocoagulation of communicating vessels in severe twin-twin transfusion syndrome in women with an anterior placenta. Obstet Gynecol 2001; 97:477-81.

7. Valsky DV, Eixarch E, Martinez JM, Crispi F, Gratacós E. Selective intrauterine growth restriction in monochorionic twins: pathophysiology, diagnostic approach and management dilemmas. Semin Fetal Neonatal Med 2010; 15:342-8.

8. Valsky DV, Eixarch E, Martinez JM, Gratacós E. Selective intrauterine growth restriction in monochorionic diamniotic twin pregnancies. Prenat Diagn 2010; 30:719-26.

9. Gratacós E, Ortiz JU, Martinez JM. A systematic approach to the differential diagnosis and management of the complications of monochorionic twin pregnancies. Fetal Diagn Ther 2012; 32:145-55

10. Walsh CA, McAuliffe FM. Recurrent twin-twin transfusion syndrome after selective fetoscopic laser photocoagulation: a systematic review of the literature. Ultrasound Obstet Gynecol 2012; 40:506-12.

11. De Lia JE, Cruikshank DP, Keye WR Jr. Fetoscopic neodymium: YAG laser occlusion of placenta vessels in severe twin-twin transfusion syndrome. Obstet Gynecol 1990; 75:1046-53.

12. De Lia JE, Kuhlmann RS, Harstad TW, Cruikshank DP. Fetoscopic laser ablation of placental vessels in severe previable twin-twin transfusion syndrome. Am J Obstet Gynecol 1995; 172(4 Pt 1):1202-8.

13. Ville Y, Hyett J, Hecher K, Nicolaides K. Preliminary experience with endoscopic laser surgery for severe twin-twin transfusion syndrome. New Engl J Med 1995; 332: 224-7.

14. Ville Y, Hecher K, Gagnon A, et al. Endoscopic laser coagulation in the management of severe twin-to-twin transfusion syndrome. Br J Obstet Gynaecol 1998; 105:446-53.

15. Roberts D, Gates S, Kilby M, Neilson JP. Interventions for twin-twin transfusion syndrome: a Cochrane review. Ultrasound Obstet Gynecol 2008; 31:701-11. 
16. Mari G, Roberts A, Detti L, et al. Perinatal morbidity and mortality rates in severe twin-twin transfusion syndrome: results of the International Amnioreduction Registry. Am J Obstet Gynecol 2001; 185:708-15.

17. Taylor MJ, Shalev E, Tanawattanacharoen S, et al. Ultrasound-guided umbilica cord occlusion using bipolar diathermy for Stage III/IV twin-twin transfusion syndrome. Prenat Diagn 2002; 22:70-6.

18. Saade GR, Belfort MA, Berry DL, et al. Amniotic septostomy for the treatmen of twin oligohydramnios-polyhydramnios sequence. Fetal Diagn Ther 1998 13:86-93.

19. Saade G, Moise K, Dorman K, et al. A randomized trial of septostomy versus amnioreduction in the treatment of twin oligohydramnios polyhydramnios sequence (TOPS). Am J ObstetGynecol 2002; 187:S54. Abstract 3.

20. Hecher K, Plath H, Bregenzer T, Hansmann M, Hackeloer BJ. Endoscopic laser surgery versus serial amniocenteses in the treatment of severe twin-twin transfusion syndrome (Abstract). 23rd Annual Meeting of the Society for Maternal-Fetal Medicine. San Francisco, USA, 2003 February 3-8.

21. Quintero RA, Comas C, Bornick PW, Allen MH, Kruger M. Selective versus nonselective laser photocoagulation of placental vessels in twin-to-twin transfusion syndrome. Ultrasound Obstet Gynecol 2000; 16:230-6.

22. Duncombe GJ, Dickinson JE, Evans SF. Perinatal characteristics and outcomes of pregnancies complicated by twin-twin transfusion syndrome. Obstet Gynecol 2003; 101:1190-6.

23. Nicolini U, Poblete A. Single intrauterine death in monochorionic twin pregnancies. Ultrasound Obstet Gynecol 1999; 14:297-301.

24. Müllers SM, McAuliffe FM, Kent E, et al. Outcome following selective fetoscopic laser ablation for twin to twin transfusion syndrome: an 8 year national collaborative experience. Eur J Obstet Gynecol Reprod Biol 2015; 191:125-9. 